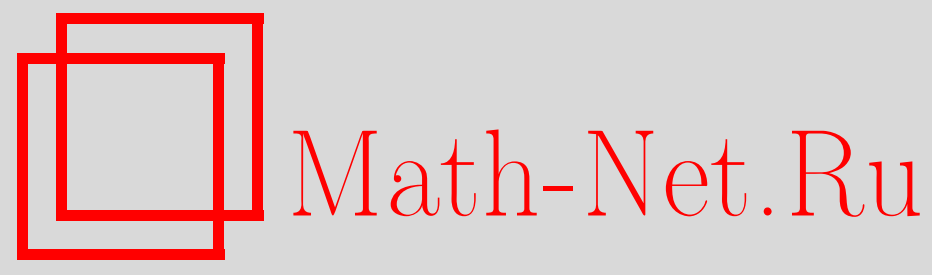

А. Н. Максименко, Многогранники коммивояжера и разрезов. Аффинная сводимость, Дискрет. матем., 2013, том 25, выпуск 2, 31-38

DOI: https://doi.org/10.4213/dm1232

Использование Общероссийского математического портала Math-Net.Ru подразумевает, что вы прочитали и согласны с пользовательским соглашением http://www.mathnet.ru/rus/agreement

Параметры загрузки:

IP : 54.174 .149 .18

26 апреля 2023 г., 13:57:44

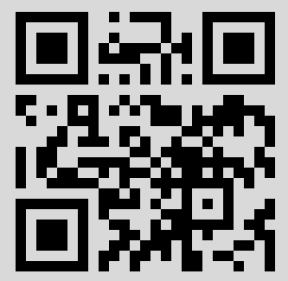




\title{
Многогранники коммивояжера и разрезов. Аффинная сводимость
}

\author{
(c) 2013 г. А. Н. Максименко
}

\begin{abstract}
Пусть $S T S P_{m}$ - многогранник задачи коммивояжера для $m$ городов, а $C U T_{n}$ - многогранник разрезов полного графа на $n$ вершинах. Показано, что $C U T_{n}$ аффинно эквивалентен одной из граней многогранника $S T S P_{m}$ при $m=(2 n-2)(2 n-3)$. С другой стороны, $S T S P_{m}$ является аффинным образом одной из граней многогранника $C U T_{n}$ при $n=(m-1)^{2}+1$.
\end{abstract}

Благодаря своей широкой известности задача коммивояжера вот уже более полувека являет собой образец труднорешаемой задачи, на котором тестируются новые идеи и методики дискретной оптимизации. Наиболее свежие результаты можно найти в монографии [1], целиком посвященной этой задаче. Другой $N P$-трудной задачей, претендующей на роль лидера по числу посвященных ей публикаций, является задача о максимальном разрезе [2]. Эта задача выгодно отличается от других труднорешаемых задач (в том числе и от задачи коммивояжера) простой формулировкой, делающей ее одной из самых «удобных» для исследований. Современные успехи в построении эффективных алгоритмов решения этих задач опираются на исследования ассоциированных с ними многогранников. С течением времени был разработан ряд общих подходов к изучению таких многогранников. Но, как правило, многогранники разных задач исследовались отдельно и, часто, независимо друг от друга. При сравнении получающихся результатов обнаруживались общие свойства. Например, суперполиномиальное кликовое число графа многогранника [3] и суперэкспоненциальное число фасет $[2,1]$. Ниже раскрывается природа этих совпадений.

Введем следующие обозначения. Для каждого гамильтонова контура в полном ориентированном графе $G$ на $k$ вершинах рассмотрим его характеристический вектор в пространстве $R^{d}$, где $d=k(k-1)$ - число дуг графа $G$. Выпуклая оболочка множества всех таких векторов называется многогранником асимметричной задачи коммивояжера. Обозначим его $A T S P_{k}$. Рассматривая различные значения $k \geqslant 3$ можно получить целое семейство таких многогранников, которое обозначим ATSP.

Аналогично определяется многогранник симметричной задачи коммивояжера. Его множество вершин образуют характеристические векторы всех гамильтоновых циклов в полном неориентированном графе $\hat{G}$ на $m$ вершинах. Обозначим этот многогранник $S T S P_{m}$, а семейство многогранников - STSP.

Те же соображения используются для определения многогранника разрезов. Рассмотрим полный неориентированный граф $H$ на $n$ вершинах. Каждое подмножество $S \subseteq V$ его вершин, включая пустое множество, однозначно определяет разрез графа 
$H$ как множество всех ребер, один конец которых лежит в $S$, а другой - в $V \backslash S$. Характеристические векторы всех $2^{n-1}$ таких разрезов определяют множество вершин многогранника разрезов $C U T_{n}$. Семейство всех таких многогранников при $n \geqslant 2$ обозначаем $C U T$.

Похожие рассуждения приводят к построению многогранников многих других задач комбинаторной оптимизации. Для двух таких произвольно выбранных задач рассмотрим их семейства многогранников $P$ и $Q$.

Определение 1. Будем говорить, что семейство многогранников $P$ афбфинно сводится к семейству $Q$, если для каждого $P_{n} \in P$ существует такой $Q_{k} \in Q$, что $P_{n}$ является аффинным образом некоторой грани (или всего многогранника) $Q_{k}$. При этом размерность последнего полиномиально зависит от размерности $P_{n}$. Обозначение: $P \propto_{A} Q$.

Такая терминология позволяет кратко сформулировать основные результаты статьи: $C U T \propto_{A} A T S P$ и $A T S P \propto_{A} C U T$. Но прежде разберем подробно более простой пример аффинной сводимости.

\section{1. Симметричная и асимметричная задачи комми- вояжера}

О сходстве многогранников $S T S P$ и $A T S P$ было известно и раньше (см., например, $[5,6])$, ниже этот факт лишь приобретает новый вид. Основная же цель этого раздела - дать возможность читателю познакомиться с теми рассуждениями, которые ниже будут использованы для более сложных конструкций.

Теорема 1. Многогранник $A T S P_{k}$ афбинно эквивалентен грани многогранника $S T S P_{m}$ для $m=2 k$.

Доказательство. Как и прежде, через $G$ обозначаем ориентированный граф асимметричной задачи, а через $V=\left\{v_{1}, v_{2}, \ldots, v_{k}\right\}-$ множество его вершин. Через $x_{i j}$ обозначим координату пространства $\mathbb{R}^{k(k-1)}$, соответствующую дуге $\left(v_{i}, v_{j}\right)$, $1 \leqslant i, j \leqslant k, i \neq j$. Положим $m=2 k$ и введем похожие обозначения для аналогичной симметричной задачи. Множество вершин неориентированного графа $\hat{G}$ обозначаем $W=\left\{w_{1}, w_{2}, \ldots, w_{m}\right\}$, а через $y_{i j}$ обозначим координату пространства $\mathbb{R}^{m(m-1) / 2}$, соответствующую ребру $\left(w_{i}, w_{j}\right), 1 \leqslant i<j \leqslant m$.

Заметим, что гиперплоскости вида $y_{i j}=0$ и $y_{i j}=1$ являются опорными для многогранника $S T S P_{m}$ (пересечение такой гиперплоскости и многогранника является гранью многогранника). Кроме того, гиперплоскости $y_{i j}=0$ и $y_{i j}=1$ разбивают множество вершин многогранника $S T S P_{m}$ на два подмножества, соответствующие гамильтоновым циклам, содержащим или же не содержащим ребро $\left(w_{i}, w_{j}\right)$. Этой особенностью воспользуемся, чтобы определить грань, аффинно эквивалентную многограннику $A T S P_{k}$.

Разобьем множество вершин $W$ на два равномощных подмножества $T=$ $\left\{t_{1}, t_{2}, \ldots, t_{k}\right\}$ и $U=\left\{u_{1}, u_{2}, \ldots, u_{k}\right\}$. Рассмотрим набор $S$ гамильтоновых циклов, каждый из которых обладает следующими свойствами:

(1) Не проходит по ребрам вида $\left(t_{i}, t_{j}\right)$ и $\left(u_{i}, u_{j}\right), 1 \leqslant i<j \leqslant k$.

(2) Обязательно содержит ребра $\left(t_{i}, u_{i}\right), 1 \leqslant i \leqslant k$. 
В силу сделанного выше замечания эти циклы соответствуют множеству вершин некоторой грани многогранника $S T S P_{m}$. Обозначим ее $\tilde{S}$. Заметим, что любой гамильтонов цикл из набора $S$ кроме $k$ «обязательных» ребер должен содержать еще $k$ ребер вида $\left(t_{i}, u_{j}\right), 1 \leqslant i, j \leqslant k, i \neq j$. Поставим в соответствие каждому ребру $\left(t_{i}, u_{j}\right)$ графа $\hat{G}$ дугу $\left(v_{i}, v_{j}\right)$ графа $G$ асимметричной задачи. Нетрудно убедиться, что таким образом будет установлено взаимно-однозначное соответствие между гамильтоновыми циклами набора $S$ и гамильтоновыми контурами графа $G$. Отсюда следует, что грань $\tilde{S}$ многогранника $S T S P_{m}$ аффинно эквивалентна многограннику $A T S P_{k}$.

С другой стороны, многогранник $A T S P_{k}$ может быть преобразован в $S T S P_{m}$, где $m=k$, с помощью очевидного линейного отображения: $y_{i j}=x_{i j}+x_{j i}, 1 \leqslant i<j \leqslant$ $k$. При этом каждая пара противоположно направленных гамильтоновых контуров будет переводиться в один гамильтонов цикл.

Таким образом,

$$
A T S P \propto_{A} S T S P \quad \text { и } S T S P \propto_{A} A T S P .
$$

Ниже все рассмотрения будут касаться лишь многогранника асимметричной задачи. Теперь они могут быть легко перенесены и на симметричный случай.

\section{2. Многогранник разрезов является гранью много- гранника коммивояжера}

Теорема 2. Многогранник $C U T_{n}$ аффинно эквивалентен грани многогранника $A T S P_{k}$, где $k=(n-1)(2 n-3)$.

Доказательство. В общих чертах искомая грань будет определяться следующим образом. Ее вершинами могут быть те и только те гамильтоновы контуры, которые целиком лежат внутри описываемого ниже подграфа $G^{\prime}$ графа $G$. Т.е. грань эта будет лежать в пересечении гиперплоскостей вида $x_{i j}=0$, где координата $x_{i j}$ соответствует дуге, не входящей в подграф $G^{\prime}$.

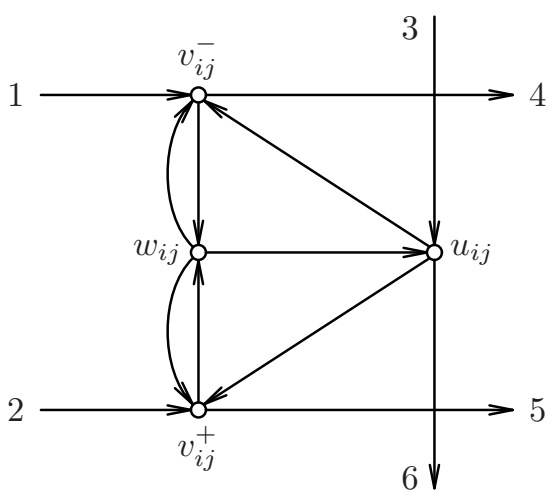

Рис. 1. Компонента $E_{i j}$.

2 Дискретная математика, т.25 №2 
Построения начнем с описания свойств типичной компоненты $E_{i j}$ (см. рис. 1) графа $G^{\prime}, 1 \leqslant i<j \leqslant n-1$. Эта компонента состоит из четырех вершин, для удобства обозначенных $v_{i j}^{-}, v_{i j}^{+}, w_{i j}$ и $u_{i j}$. Все дуги графа $G^{\prime}$, инцидентные этим вершинам, указаны на рисунке. Как видно, вершина $w_{i j}$ не имеет непосредственных «соединений» с внешними для компоненты $E_{i j}$ вершинами.

$\{1\}$

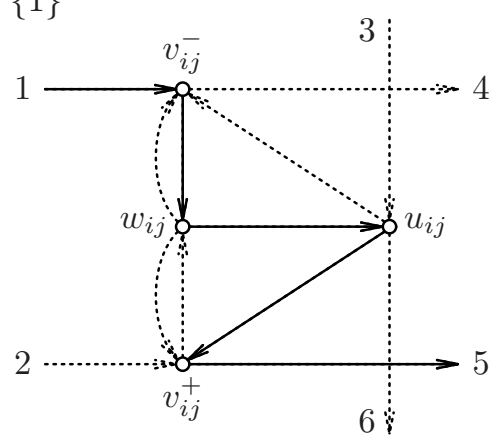

$\{1,3\}$

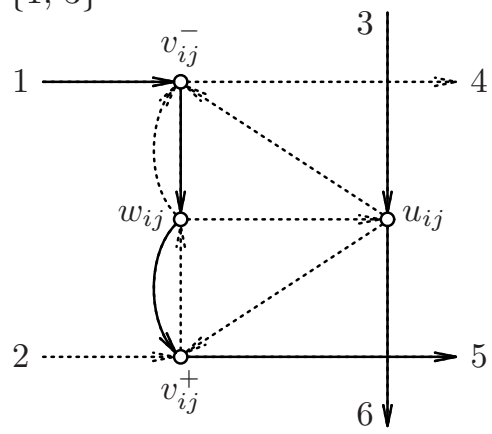

$\{2\}$

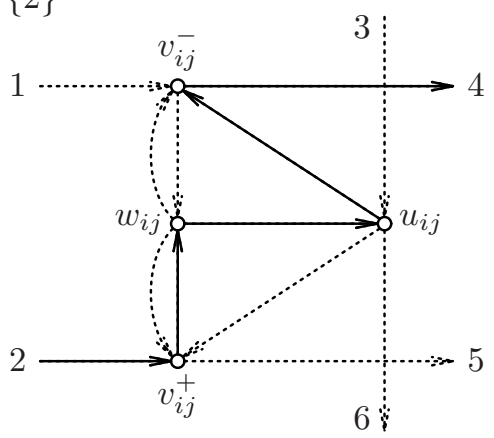

$\{2,3\}$

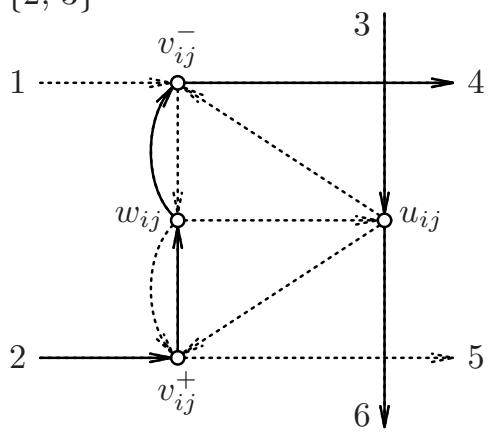

Рис. 2. Гамильтонов контур в компоненте $E_{i j}$.

Будем предполагать, кроме того, что проходящий через эту компоненту гамильтонов контур обязательно содержит ровно одну из дуг 1 и 2 , оканчивающихся в вершинах $v_{i j}^{-}$и $v_{i j}^{+}$, соответственно. Нетрудно проверить, что такой гамильтонов контур может иметь лишь одну из четырех конфигураций, представленных на рис. 2. При этом набор дуг каждой конфигурации однозначно определяется набором направленных извне в компоненту дуг 1, 2 и 3.

Чтобы убедиться в этом, введем следующие обозначения. Координаты вектора в $\mathbb{R}^{k}$, соответствующие дугам $1-6$, обозначим $x_{i j}^{1}, \ldots, x_{i j}^{6}$ соответственно. Для произвольного гамильтонова контура они могут принимать только значения 0 или 1. Так как гамильтонов контур должен содержать ровно одну из дуг 1 и 2, получаем первое уравнение

$$
x_{i j}^{1}=1-x_{i j}^{2}
$$

На рис. 2 можно углядеть еще ряд соотношений:

$$
x_{i j}^{5}=1-x_{i j}^{2}, \quad x_{i j}^{4}=x_{i j}^{2}, \quad x_{i j}^{6}=x_{i j}^{3} .
$$


Теперь разберемся с внутренними дугами компоненты. Для произвольной дуги, ведущей из вершины $a$ в вершину $b$, соответствующую координату характеристического вектора обозначаем $x(a, b)$. Из рис. 2 видны следующие взаимосвязи:

$$
x\left(v_{i j}^{+}, w_{i j}\right)=x_{i j}^{2}, \quad x\left(v_{i j}^{-}, w_{i j}\right)=1-x_{i j}^{2}, \quad x\left(w_{i j}, u_{i j}\right)=1-x_{i j}^{3} .
$$

Для характеризации оставшихся четырех дуг введем дополнительную переменную

$$
e_{i j}=x_{i j}^{2} \oplus x_{i j}^{3} \quad \text { (сложение по модулю 2). }
$$

$\mathrm{C}$ ее использованием оставшиеся уравнения приобретают вид

$$
\begin{array}{llrl}
x\left(u_{i j}, v_{i j}^{-}\right) & =\frac{1}{2}\left(e_{i j}+x_{i j}^{2}-x_{i j}^{3}\right), & & x\left(w_{i j}, v_{i j}^{+}\right)=\frac{1}{2}\left(e_{i j}+x_{i j}^{3}-x_{i j}^{2}\right), \\
x\left(u_{i j}, v_{i j}^{+}\right)=1-\frac{1}{2}\left(e_{i j}+x_{i j}^{2}+x_{i j}^{3}\right), & x\left(w_{i j}, v_{i j}^{-}\right)=\frac{1}{2}\left(x_{i j}^{2}+x_{i j}^{3}-e_{i j}\right) .
\end{array}
$$

Итак, компонента построена таким образом, что присутствие любой ее дуги в гамильтоновом контуре линейно зависит лишь от присутствия дуг 2 и 3 , и дополнительной переменной $e_{i j}$.

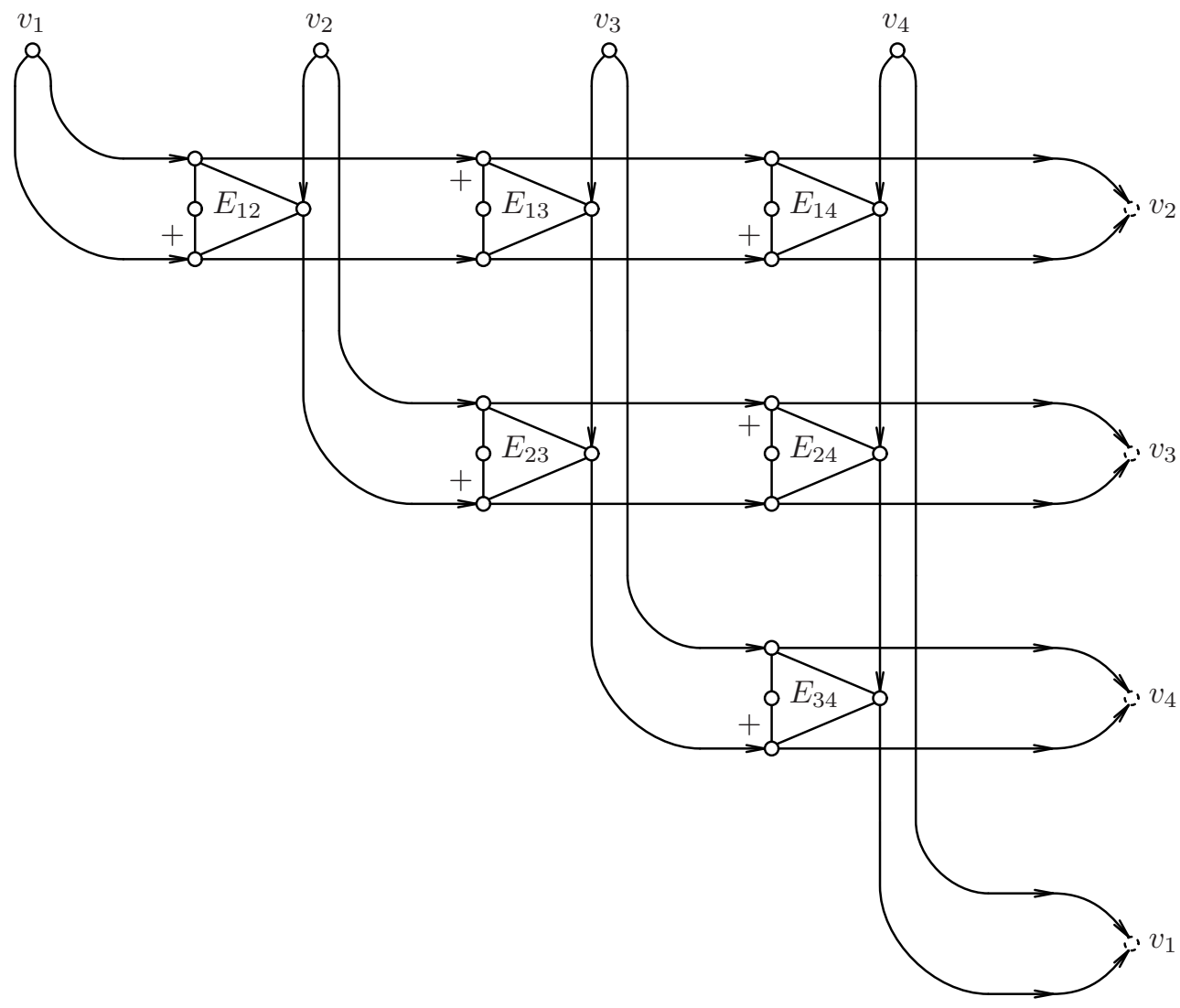

Рис. 3. Подграф $G^{\prime}$ для $n=5$.

Теперь приступим к описанию всего подграфа $G^{\prime}$. Он будет состоять из компонент $E_{i j}, 1 \leqslant i<j \leqslant n-1$, и дополнительных вершин $v_{i}, 1 \leqslant i \leqslant n-1$. Каждая 
компонента соединяется своими внешними дугами с «соседними» по индексам компонентами, а «крайние» компоненты соединяются с дополнительными вершинами так, как это показано на рис. 3. Из каждой дополнительной вершины $v_{i}$ выходит ровно две дуги. Заметим, что любой произвольно выбранный гамильтонов контур нашего подграфа должен содержать ровно одну из них. Граф сконструирован таким образом, что конфигурация любого его гамильтонова контура однозначно определяется набором выходящих из дополнительных вершин дуг. Таким образом число всех гамильтоновых контуров равно $2^{n-1}$ и совпадает с числом всевозможных разрезов графа $H$ для задачи о разрезе. Остается определить линейные соотношения, связывающие дуги графа $G^{\prime}$ и ребра графа $H$.

Обозначим через $y_{i j}$ координату пространства $\mathbb{R}^{n(n-1) / 2}$, характеризующую наличие ребра графа $H$, соединяющего $i$-ю и $j$-ю вершины, $1 \leqslant i, j \leqslant n$. Для дуги $(a, b)$ графа $G^{\prime}$ соответствующую координату обозначаем $x(a, b)$. Положим

$$
\begin{array}{rlrl}
x\left(v_{1}, v_{1,2}^{+}\right) & =y_{1, n}, & & x\left(v_{1}, v_{1,2}^{-}\right)=1-y_{1, n}, \\
x\left(v_{2}, u_{1,2}\right) & =y_{2, n}, & & x\left(v_{2}, v_{2,3}^{-}\right)=1-y_{2, n}, \\
x\left(v_{3}, u_{1,3}\right) & =y_{3, n}, & & x\left(v_{3}, v_{3,4}^{-}\right)=1-y_{3, n}, \\
& \cdots \cdots \\
& \cdots & & \cdots \\
x\left(v_{n-1}, u_{1, n-1}\right) & =y_{n-1, n}, & & x\left(v_{n-1}, v_{1}\right)=1-y_{n-1, n} .
\end{array}
$$

Из уравнений $(2)$, с учетом конструкции всего графа $G^{\prime}$, получаем $x_{i j}^{2}=y_{i, n}$ и $x_{i j}^{3}=y_{j, n}$. Дополнительную переменную $e_{i j}$, ранее определявшуюся уравнением (4), положим равной $y_{i j}$. Ясно, что это будет эквивалентная замена. Все остальные координаты определяются линейными неоднородными уравнениями (1)-(3), (5).

Таким образом, указанный граф $G^{\prime}$ определяет грань многогранника $A T S P_{k}$, где $k=(n-1)(2 n-3)$, аффинно эквивалентную многограннику $C U T_{n}$.

\section{ATSP аффинно сводится к CUT}

Подобрать грань многогранника разрезов, которая была бы аффинно эквивалентна $A T S P_{k}$, для наперед заданного $k \geqslant 6$ не удастся. Дело в том, что многогранники разрезов 2-смежностны (см., например, $[2,3])$. Следовательно, 2-смежностны и все их грани. В то же время многогранник $A T S P_{k}$ при $k \geqslant 6$ не 2-смежностен [7].

Тем не менее, у нас остается возможность указать вырожденное аффинное отображение, переводящее грань многогранника разрезов в многогранник коммивояжеpa.

Теорема 3. Многогранник $A T S P_{k}$ является образом вырожденного афбинного отображения некоторой грани многогранника $C U T_{n}$, где $n=(k-1)^{2}+1$.

Доказательство. Положим $n=(k-1)^{2}+1$ и вершины графа $H$ задачи о разрезе будем обозначать $w_{i j}(1 \leqslant i, j \leqslant k-1)$. Их удобно представлять в виде ячеек квадратной матрицы. «Последнюю» вершину обозначим $w_{n}$, она будет располагаться вне матрицы.

Крайние точки интересующей нас грани будут соответствовать разрезам следующего вида. Одно из двух подмножеств вершин графа $H$, определяющее такой разрез, должно состоять из $k-1$ ячеек указанной матрицы, никакие две из которых не лежат ни в одной строке, ни в одном столбце. Ясно, что число таких разрезов 
будет совпадать с числом всех гамильтоновых контуров орграфа $G$ на $k$ вершинах. Нетрудно также указать вырожденное аффинное отображение, переводящее указанные разрезы в гамильтоновы контура. Значение координаты $y_{k j}, 1 \leqslant j<k$, соответствующей дуге из $k$-й («последней») в $j$-ю вершину графа $G$, будет совпадать со значением координаты $x\left(w_{1 j}, w_{n}\right)$, соответствующей ребру $\left(w_{1 j}, w_{n}\right)$ графа $H$. Координату $y_{j k}, 1 \leqslant j<k$, положим равной $x\left(w_{k-1, j}, w_{n}\right)$. И, наконец, для произвольных $1 \leqslant i, j \leqslant k-1, i \neq j$, положим

$$
y_{i j}=\frac{1}{2} \sum_{l=1}^{k-2}\left(x\left(w_{l i}, w_{n}\right)+x\left(w_{l+1, j}, w_{n}\right)-x\left(w_{l i}, w_{l+1, j}\right)\right) .
$$

Заметим, что выражение $x\left(w_{l i}, w_{n}\right)+x\left(w_{l+1, j}, w_{n}\right)-x\left(w_{l i}, w_{l+1, j}\right)$ отличается от нуля только в том случае, когда пара вершин $w_{l i}$ и $w_{l+1, j}$ отделена разрезом от вершины $w_{n}$.

Остается доказать, что указанное множество разрезов действительно образует грань многогранника $C U T_{n}$. Для этого определим уравнение опорной для многогранника $C U T_{n}$ гиперплоскости $F$ такой, что в пересечении $C U T_{n} \cap F$ лежат все интересующие нас разрезы и только они. Выделим из всех ребер графа $H$ два подмножества $X$ и $Y$. В подмножество $X$ будут входить все те ребра, обе вершины которых лежат либо в одной строке, либо в одном столбце указанной матрицы. Подмножество $Y$ будет состоять из всех ребер, инцидентных $w_{n}$. Координаты направляющего вектора $a$ опорной гиперплоскости положим равными 1 для всех ребер из множества $X$, равными $(5-2 k)$ для ребер множества $Y$ и равными 0 для всех остальных ребер. Тогда скалярное произведение $(a, x)$, где $x$ выбирается из множества вершин многогранника $C U T_{n}$, будет достигать своего максимального значения $k-1$ для всех разрезов указанного вида и только для них.

\section{4. Заключение}

По аналогии с теорией полиномиальной сводимости $N P$-полных задач для многогранников коммивояжера и разрезов удалось доказать следующие цепочки утверждений:

$$
C U T \propto_{A} A T S P \propto_{A} S T S P \quad \text { и } \quad S T S P \propto_{A} A T S P \propto_{A} C U T .
$$

Конструктивность этих сведе́ний позволяет проецировать внешнее (линейное) описание любого из упомянутых выше многогранников на описания остальных. Обнаруженная взаимосвязь вскрывает причины сходства некоторых свойств многогранников коммивояжера и разрезных многогранников. Так, в частности, на основании того, что граф многогранника $C U T_{n}$ полный, теперь нетрудно сделать заключение о том, что граф многогранника $A T S P_{k}$ содержит полный подграф как минимум на $2 \sqrt{k / 2}-1$ вершинах. Более того, из теоремы 2 и свойства 3 -смежностности многогранника $C U T_{n}[2]$ следует, что $A T S P_{k}$ содержит 3-смежностную грань с суперполиномиальным числом $2^{\sqrt{k / 2}-1}$ вершин. Тем не менее, открытым остается следующий вопрос. Верно ли, что для любого $p \in \mathbb{N}$ найдется номер $k$, начиная с которого $A T S P_{k}$ содержит $p$-смежностную грань с суперполиномиальным (относительно $k$ ) числом вершин? 


\section{Список литературы}

1. Applegate D.L., Bixby R.E., Chvatal V., Cook W.J., The Traveling Salesman Problem: A Computational Study. Princeton University Press, 2006.

2. Деза М. М., Лоран М., Геометрия разрезов и метрик. МЦНМО, Москва, 2001.

3. Бондаренко В.А., Максименко А.Н., Геометрические конструкиии и сложность в комбинаторной оптимизачии. ЛКИ, Москва, 2008.

4. Гэри М., Джонсон Д., Вычислительные машины и труднорешаемые задачи. Мир, Москва, 1982.

5. Junger M., Reinelt G. and Rinaldi G., The Traveling Salesman Problem // Handbooks in Operations Research and Management Science, 7. Elsevier, Amsterdam, 1995.

6. Бондаренко В. А., Полиэдральные графы и сложность в комбинаторной оптимизации. ЯрГУ, Ярославль, 1995.

7. Padberg M.W., Rao M.R., The travelling salesman problem and a class of polyhedra of diameter two. Math. Prog. (1974) 7, №1, 32-45.

8. Бондаренко В. А., Неполиномиальная нижняя оценка сложности задачи коммивояжера в одном классе алгоритмов. Автоматика и телемеханика (1983) 9, 45-50.

9. Papadimitriou C. H., The adjacency relation on the traveling salesman polytope is NPcomplete. Math. Prog. (1978) 14, №1, 312-324.

Статья поступила 09.09.2009. 